\title{
XPO1 Inhibitor SL-801
}

National Cancer Institute

\section{Source}

National Cancer Institute. XPO1 Inhibitor SL-801. NCI Thesaurus. Code C128246.

An orally bioavailable inhibitor of the nuclear export protein exportin-1 (XPO1;

chromosome region maintenance 1 protein homolog; CRM1), with potential

antineoplastic and pro-apoptotic activities. Upon administration, XPO1 inhibitor SL-801

reversibly binds to the cargo binding site of XPO1, and prevents the XPO1-mediated nuclear export of cargo proteins, including tumor suppressor proteins (TSPs), such as

p53, FOXO, p21, and p27, and leads to their selective accumulation in the nuclei of tumor cells. As a selective inhibitor of nuclear export (SINE), SL-801 restores the nuclear localization and function of TSPs, which leads to the induction of apoptosis in tumor cells. XPO1, the major export factor that transports proteins and RNA from the nucleus to the cytoplasm, is overexpressed in a variety of cancer cell types while minimally expressed in normal, healthy cells. The dysregulated export of TSPs into the cytoplasm prevents TSPinitiated apoptosis. XPO1 overexpression leads to uncontrolled tumor cell proliferation and is associated with poor prognosis. 